\title{
Performance evaluation of an in-house human immunodeficiency virus type-1 protease-reverse transcriptase genotyping assay in Cameroon
}

\author{
J. Fokam $\cdot$ R. Salpini $\cdot$ M. M. Santoro $\cdot$ V. Cento $\cdot$ \\ R. D'Arrigo · C. Gori · C. F. Perno $\cdot$ V. Colizzi · \\ A. Nanfack - L. C. Gwom - G. Cappelli - D. Takou
}

Received: 17 September 2010/ Accepted: 14 March 2011

(C) Springer-Verlag 2011

\begin{abstract}
Most commercial HIV-1 genotyping assays are hampered by high cost in resource-limited settings. Moreover, their performance might be influenced over time by HIV genetic heterogeneity and evolution. An in-house genotyping protocol was developed, and its sequencing performance and reproducibility were compared to that of ViroSeq ${ }^{\mathrm{TM}}$. One hundred ninety plasma samples from HIV-1-infected subjects in Cameroon, a resource-limited setting with a high HIV genetic variability, were processed for pol gene sequencing with an in-house protocol, ViroSeq $^{\mathrm{TM}}$, or both. Only non-B subtypes were found. The in-house sequencing performance was $98.7 \%$ against $92.1 \%$ with ViroSeq ${ }^{\mathrm{TM}}$. Among 36 sequence pairs obtained using both assays, the overall rate of discordant amino acid
\end{abstract}

J. Fokam and R. Salpini contributed equally to this work.

J. Fokam - V. Colizzi - A. Nanfack - L. C. Gwom ·

G. Cappelli · D. Takou

CIRCB: Chantal BIYA International Research Centre

for Research on HIV/AIDS Prevention and Management,

Yaoundé, Cameroon

J. Fokam - L. C. Gwom

Faculty of Medicine and Biomedical Sciences,

University of Yaoundé, Yaoundé, Cameroon

R. Salpini $(\bowtie) \cdot$ M. M. Santoro · V. Cento ·

C. F. Perno - V. Colizzi

Department of Experimental Medicine and Biochemical

Sciences, University of Rome Tor Vergata, Via Montpellier, 1,

00133 Rome, Italy

e-mail: rsalpini@yahoo.it

R. D’Arrigo · C. Gori · C. F. Perno

IRCSS L Spallanzani, Rome, Italy

G. Cappelli

National Council of Research, Rome, Italy positions was negligible $(0.24 \%)$. With its high sensitivity and reproducibility, as well as its affordable cost (about half of ViroSeq ${ }^{\text {TM: }} 92 €$ vs. $217 €$ ), this in-house assay is a suitable alternative for HIV-1 genotyping in resource-limited and/or in high-genetic-diversity settings.

\section{Introduction}

Human immunodeficiency virus (HIV) infection remains a major global health priority, with about 33.4 millions [31.1-35.8] people infected worldwide [1]. Even though access to antiretroviral therapy has helped in decreasing its morbidity and mortality, the rate of HIV-1 infection is still very high in sub-Saharan Africa (70\%), where access to appropriate healthcare is limited by socio-economical factors [2-4]. With the increasing treatment possibilities, the evaluation of transmitted and acquired strains that are resistant to available antiviral drugs has become essential for sub-Saharan countries [5]. HIV-1 genotypic drug resistance testing (GRT) is considered an important basic tool to guide the choice of antiretroviral therapy in the European and North American countries. For this reason, the guidelines recommend genotypic resistance testing both in antiretroviral naïve patients and in patients with suboptimal virological responses or virologic failure $[6,7]$.

By contrast, the World Health Organization (WHO) recommends the performance of GRT only for the surveillance of transmitted and acquired drug resistance in resource-limited settings. [5] Although patients in Cameroon have been receiving free highly active antiretroviral therapy (HAART) since 2007, the management of HIV infection within the health care system is still of poor quality due to insufficient and generic treatment guidelines, financial restrictions to individual patient follow-up and 
setbacks in diagnostics due to the high viral genetic variability $[8,9]$. Cameroon is a setting where all of the known groups of HIV-1, the rare group O (Outlier), group N (Non$\mathrm{O}$, Non-M), and the recent group $\mathrm{P}$, have been identified from people with epidemiologic links to this country [1012]. Therefore, this country is a field setting with features that are suitable for assessing the performance of a genotyping tool that could be useful for HIV-1 GRT at an affordable cost, in order to improve the management of infected patients in health systems with similar characteristics. HIV-1 epidemiology in this country is reported to be stable, from $5.5 \%$ in 2004 to an estimate of $5.1 \%$ in 2009 , for a total of 558,228 people living with HIV/acquired immunodeficiency deficiency syndrome (AIDS). With a rapid scaling-up to antiretroviral therapy (1.3\% in 2003 to $50 \%$ in 2009), at least 76,228 patients are under HAART, including 3,114 children and 51,032 women in the entire national territory [13]. In this context, drug resistance testing is gradually becoming an indispensable tool for the surveillance and prevention of emerging resistant viral strains as well as for individualised patient monitoring [5, 14]. Most genotyping methods detect HIV drug resistance by nucleic acid sequencing in the protease (PR) and reverse transcriptase (RT) regions of the pol gene [15-17]. Two genotyping methods, approved by the Food and Drug Administration (FDA), ViroSeq ${ }^{\mathrm{TM}}$ HIV-1 Genotyping Version 2.7 from Celera Diagnostics, Alameda, CA, USA [18-20], and TruGene [21] are currently available commercially. Interestingly, it should be considered that viral dynamics (due to the high error rate of RT), evolution over time (due to the high levels of HIV-1 replication), and the large spread of non-B subtypes might influence the overall performance of the existing genotyping tools [22]. Improved sensitivity of in-house sequencing assays compared to these commercially available kits has been reported by several laboratories, especially for some non-B subtypes [23-25]. These established in-house genotyping assays were designed in order to overcome cost and subtype-specific constraints [26, 27]. However, the performance of these in-house assays has not been validated against internationally approved reference systems [28, 29], except for two recent ones, developed for subtypes B, $\mathrm{C}$, and D circulating in India, and subtype C circulating in West and South Africa [24, 25].

The main findings regarding HAART (highly active antiretroviral therapy) resistance in Cameroon were generally based on the ViroSeq ${ }^{\mathrm{TM}}$ genotyping system [30-32], with the exception of an affordable in-house assay proven to be sensitive for the combined detection of plasma HIV-1 and assessment of drug resistance. Nevertheless, this inhouse method has never been compared to FDA-approved assays in terms of sequencing performance and the reproducibility of its mutation profile [27]. For the above reasons, the aim of this work was to develop and validate a reproducible, highly sensitive and affordable genotyping protocol for drug resistance surveillance and/or individualized monitoring in a context of high viral genetic diversity and limited resources. This would significantly improve the clinical management of patients infected with genetically different HIV-1 strains living in the sub-Saharan areas as well as in other developing countries.

\section{Materials and methods}

\section{Study population}

One hundred ninety plasma samples were collected from different HIV-1-infected Cameroonian individuals from March 2008 to February 2010 and analyzed at the Molecular Microbiology Unit of Chantal BIYA International Reference Centre for research (CIRCB) on HIV/ AIDS prevention and management in Yaoundé, Cameroon. Of the overall population, $56.9 \%$ (108) were children, and $43.1 \%$ (82) were adults, with a mean age of 7.25 years [min-max: 4 months-17 years] and 40.47 years [2568 years], respectively. The number of males and females was similar (90 [47.4\%] vs. 100 [52.6\%]). At the moment of samples collection, 43 (22.6\%) individuals were drugnaïve, $140(73.7 \%)$ were drug-experienced, and 7 (3.7\%) had an unknown antiretroviral therapeutic history.

All the samples were subjected to HIV-1 GRT, with the addition of ViroSeq ${ }^{\mathrm{TM}}$-positive and negative standards used as quality control for every reaction. With the exception of three samples with a viral load above the limit of detection ( $>7 \log _{10}$ RNA copies/ml), the overall mean plasma viral load was 5.67 (min-max: 1.79-6.96) $\log _{10}$ RNA copies $/ \mathrm{ml}$. Of these samples, 156/190 were processed with the in-house genotyping assay, and 76/190 with ViroSeq ${ }^{\mathrm{TM}}$. The average viremia of samples tested with each protocol was nearly identical (in-house assay: $5.70 \log _{10}$ RNA copies/ml; Viroseq ${ }^{\mathrm{TM}}$ : $5.69 \log _{10}$ RNA copies/ml; min-max for both protocols: $1.79-6.96 \log _{10}$ RNA copies/ $\mathrm{ml}$ ). Of all of the samples tested, only three indicated viremia $<3 \log _{10}$ RNA copies/ml $(1.79,2.02,2.48)$; all of them were processed with the in-house assay while two of them (viremia: 1.79, 2.48) were processed with the ViroSeq ${ }^{\mathrm{TM}}$ kit. Thirty-six samples were successfully processed by both methods and thereby used to evaluate the genotypic concordance between the two assays. The number of samples processed depended on the plasma availability in the bio-bank and on the stock of reagents available for each of the assays.

Four milliliters of whole blood was collected in ethylenediaminetetraacetic acid (EDTA) tubes. Two plasma aliquots of $1 \mathrm{ml}$ each were constituted from every blood 
sample by centrifugation at $1800 \mathrm{rpm}$ for $15 \mathrm{~min}$ and stored between +2 and $+8^{\circ} \mathrm{C}$ for 24 hours or at $-80^{\circ} \mathrm{C}$ for 6 months.

\section{RNA extraction}

Viral RNA was extracted from a one-milliliter aliquot of plasma and from the ViroSeq ${ }^{\mathrm{TM}}$-positive and negative standards using a QIAamp Viral RNA Mini Kit (QIAGEN) according to the protocol specifications. The choice of using 1 milliliter of plasma for testing instead of 500 microliters as recommended for the ViroSeq ${ }^{\mathrm{TM}}$ kit was for the purpose of increasing the amount of extracted RNA and ensuring comparable results with both assays. HIV-RNA was processed directly for reverse transcription and amplification.

\section{Reverse transcription and PCR amplification}

RNA extracts from patients and from the ViroSeq ${ }^{\mathrm{TM}}$ standards (positive and negative) were amplified using the ViroSeq ${ }^{\mathrm{TM}}$ protocol following the manufacturer's instructions (Celera Diagnostics, Alameda, CA, USA). The RNA extracts were also amplified following the developed inhouse RT polymerase chain reaction (RT-PCR) protocol. This protocol is a two-step PCR, and the primers were designed based on an analysis of conserved regions in PR and RT. Primer sequences for the first-round RT-PCR are 5'GAC AGG CTA ATT TTT TAG GG 3' as the sense primer, located at 2075-2094 ( $\mathrm{gag}$ ), and 5'TTT CCC CAT ATT ACT ATG CTT 3' as the antisense primer, located at 3683-3703 (pol) (the position is given referring to the HXB2 strain from Los Alamos National Laboratory database using the Sequence Locator and QuickAlign tools: http://www.hiv.lanl.gov/content/sequence/HIV/mainpage. html). The RT-PCR reaction was performed using a one-step kit (SuperScript ${ }^{\mathrm{TM}}$ One-Step RT-PCR System for Long Templates, Invitrogen) in a reaction containing for each sample $25 \mu$ reaction mix, $8 \mu \mathrm{lgSO}_{4}(5 \mathrm{mM}), 3 \mu \mathrm{l}$ DNAse- and RNAse-free water, $0.75 \mu \mathrm{l}$ sense primer (10 $\mu \mathrm{M}$ stock), $0.75 \mu \mathrm{l}$ antisense primer $(10 \mu \mathrm{M}$ stock), $1 \mu \mathrm{l}$ RNAseOUT (40 U/ $\mu \mathrm{l}$ Invitrogen), $1.5 \mu \mathrm{l}$ RT-Taq (Superscript ${ }^{\circledR}$ II RT/Platinum ${ }^{\circledR}$ Taq HIFI Mix) and $10 \mu \mathrm{l}$ of RNA. The RT-PCR conditions consisted of an initial step of 1 cycle at $50^{\circ} \mathrm{C}$ for $30 \mathrm{~min} ; 1$ cycle of $94^{\circ} \mathrm{C}$ for $2 \mathrm{~min}$; 40 cycles $\left(95^{\circ} \mathrm{C}, 30 \mathrm{~s} ; 51^{\circ} \mathrm{C}, 30 \mathrm{~s} ; 72^{\circ} \mathrm{C}, 90 \mathrm{~s}\right)$; a final elongation step of $72^{\circ} \mathrm{C}$ for $10 \mathrm{~min}$; and 1 cycle $4^{\circ} \mathrm{C}$ for $30 \mathrm{~min}$. The expected cDNA is about 1620 base pairs (bp) in length (position 2079 [gag]-3703 [pol]). For each PCR reaction, positive and negative controls were used to ensure the effectiveness of the reaction and the absence of cross-contamination, respectively. For samples that were insufficiently amplified after the first-round PCR, a second- round PCR (semi-nested PCR) was performed with the following primers sequences: 5' GAC AGG CTA ATT TTT TAG GG 3' as the sense primer, located at 2075-2094 (gag), and 5' GGC TCT TGA TAA ATT TGA TAT GT 3' as the antisense primer, located at 3561-3583 (pol), in a reaction containing $33.95 \mu \mathrm{l}$ DNAse- and RNAse-free water, $3 \mu \mathrm{lggl}_{2}, 0.75 \mu \mathrm{l}$ sense primer $(10 \mu \mathrm{M}$ stock $)$, $0.75 \mu \mathrm{l}$ antisense primer $(10 \mu \mathrm{M}$ stock $), 0.8 \mu \mathrm{l}$ dNTPs $(12.5 \mathrm{mM}), 5 \mu \mathrm{l}$ buffer Taq $10 \times, 0.75 \mu$ l Platinum ${ }^{\circledR}$ TaqGold $(5 \mathrm{U} / \mu \mathrm{l})$ and $5 \mu \mathrm{l}$ of cDNA. The PCR conditions were as follows: 1 cycle of $93{ }^{\circ} \mathrm{C}$ for $12 \mathrm{~min}$; 40 cycles $\left(94^{\circ} \mathrm{C}, 30 \mathrm{~s} ; 53^{\circ} \mathrm{C}, 30 \mathrm{~s} ; 72^{\circ} \mathrm{C}, 2 \mathrm{~min}\right) ; 1$ cycle of $72^{\circ} \mathrm{C}$ for $10 \mathrm{~min}$; and 1 cycle of $4^{\circ} \mathrm{C}$ for $30 \mathrm{~min}$. The expected cDNA is about $1510 \mathrm{bp}$ (position 2075 [gag] to 3583 [pol]) in length. For each semi-nested PCR reaction, positive and negative controls were used to ensure the effectiveness of the reaction and the absence of contamination, respectively.

Both the ViroSeq ${ }^{\mathrm{TM}}$ kit and the in-house protocol, which were regularly performed alongside a negative PCR control, showed no problem of cross-contamination. For the in-house protocol, an additional negative control was run for the semi-nested-PCR. PCR products were analyzed by $1 \%$ agarose gel electrophoresis by visual comparison of the amount of PCR product with a low-molecular-weight DNA ladder included in the ViroSeq ${ }^{\mathrm{TM}}$ kit. The resulting amplicon encompassing the PR-RT region (approximately 1.5 kilobases in length) was purified using a Microcon YM-100 microconcentrator (Millipore).

\section{Sequencing reaction}

The amplified products from the pol region were completely sequenced in the sense and antisense orientations using an automated sequencer (ABI 3130 Genetic Analyzer) with seven different overlapping sequence-specific primers: 5' AGC AGA CCA GAG CCA ACA GC 3'(2140$2159 \mathrm{gag}), 5$ ' CCA TCC ATT CCT GGC TTT AAT 3' (2582-2602 pol), 5' CAG GAA TGG ATG GCC CAA AA 3' (2590-2609 pol), 5' TTG TAC AGA AAT GGA AAA GGA AGG 3' (2660-2683 pol), 5' CCC TGT GGA AAG CAC ATT GTA 3' (2985-3004, with an insertion), 5' GCT TCC ACA GGG ATG GAA A 3' (2993-3011 pol), 5' CTA TTA AGT CTT TTG ATG GGT CA 3' (3506-3528 pol) (with reference to the HXB2 strain from the Los Alamos National Laboratory database using the Sequence Locator and QuickAlign tools: http://www.hiv.lanl.gov/content/ sequence/HIV/mainpage.html). These sequencing primers were designed based on the analysis of highly conserved regions among HIV-1 subtypes. The reaction mixture for the sequencing reaction contained $8 \mu \mathrm{l}$ ABI PRISM Big Dye Terminator (Perkin-Elmer), $4.8 \mu \mathrm{l}$ water, $3.2 \mu \mathrm{l}$ primer $(1 \mathrm{pmol})$ and $4 \mu \mathrm{l}$ of purified cDNA (40 ng), for a total 
volume of $20 \mu \mathrm{l}$. The sequencing conditions were as follows: 35 cycles $\left(96^{\circ} \mathrm{C}, 10 \mathrm{~s} ; 55^{\circ} \mathrm{C}, 10 \mathrm{~s} ; 60^{\circ} \mathrm{C}, 4 \mathrm{~min}\right) ; 1$ cycle of $4^{\circ} \mathrm{C}$ for $30 \mathrm{~min}$. The quality of each sequence was ensured by covering the PR-RT region with at least two sequence segments (one forward and one reverse). The sequencing product was purified by gel filtration chromatography using Sephadex G-50 resin (Sigma-Aldrich) in order to eliminate excess primers, unincorporated dideoxynucleotides (ddNTPs), and salts.

\section{Sequence analysis and detection of mutations}

Sequence data were obtained, and complete sequences encompassing the pol region of interest were assembled and manually edited using Seqscape version 2.5. All HIV-1 pol sequences $(\approx 1,197$ nucleotides) were aligned in BioEdit version 5.0.6 using CLUSTAL W [33] and compared to reference sequences for the major HIV-1 subtypes and circular recombinant forms (CRFs), available at Los Alamos database (http://www.hiv.lanl.gov). Gaps were then removed from the final alignment. The phylogenetic tree was inferred using all of the sequences generated to ensure that there was no cross-contamination of samples. Maximum-likelihood analysis for phylogenetic tree inference was performed using the PAUP* package [34]. The transversion model $(\mathrm{GTR}+\mathrm{I}+\mathrm{G})$ of nucleotide substitution was chosen using Modeltest v.3.7 implemented in PAUP* [35] as the best-fitting evolution model for tree reconstruction. The statistical robustness and reliability of the branching order within each phylogenetic tree were confirmed by a bootstrap analysis using 1000 replicates on a maximum-likelihood tree generated by PhyML [36]. Recombination among HIV-1 subtypes was assessed using SCUEAL [37], COMET [38], and SimPlot software [39, 40]. All sequences were analyzed for resistance mutations using the Stanford University HIV Drug Resistance Database (http://www.hivdb.stanford.edu). The nucleotide sequences analyzed are in the process of being submitted to GenBank.

\section{Data analysis}

The rate of successful amplification and sequencing for ViroSeq ${ }^{\mathrm{TM}}$ and the in-house method was used to evaluate the performance of the in-house assay. Drug resistance mutations in both pol regions (PR ad RT) as well as polymorphic changes in positions not related to resistance compared to HIV-1 HXB-2 as a reference strain were analyzed for each patient.

Sequences obtained by both assays were compared based on the analysis of amino acid substitutions at all positions in the PR (amino acids 1-99) and RT (amino acids 1-300) regions. The similarity between sequence pairs was evaluated by phylogenetic analysis following previously reported phylogenetic tree inference methods and by calculation of the pairwise genetic distance (estimated as the number of nucleotide substitutions per nucleotide site) in the MEGA version 4.1 package. Codons 1-99 in the PR region and 1-300 in the RT region were considered for both assays, including an overview analysis on the sequencing primer mismatch. The sequences obtained independently by both systems were analyzed for reproducibility of detection of amino acid mutations using the following criteria (as reported previously by Saravanan et al. [24]):

a. Concordant (if both assays gave the same result);

b. Partially concordant (if mixture by one assay and not by the other);

c. Discordant (if the two assays detected different amino acids).

Additionally, the same approach was used to estimate the capacity of both methods to detect drug resistance mutations. Specifically, $17 \mathrm{PR}$ and $24 \mathrm{RT}$ amino acid positions known to be related to drug resistance according to the Stanford University HIV Drug Resistance Database (http://www.hivdb.stanford.edu) were accurately analyzed for evaluation of concordance.

\section{Results}

Phylogenetic analysis

Phylogenetic analysis did not reveal any cross-contamination among the 188 samples that were successfully processed with both ViroSeq ${ }^{\mathrm{TM}}$ and the in-house assay. This analysis also revealed that CRF02_AG was the most prevalent strain (54.8\%), followed by the subtypes A1 (12.8\%), F2 (6.9\%), D (6.4\%), G (5.8\%), the recombinant CRF11 (4.3\%), and other HIV-1 non-B strains (1.6\% CRF01_ AE, $1.6 \% \mathrm{~A}, 1.1 \% \mathrm{~F} 2 / \mathrm{G}, 1.1 \% \mathrm{~F} 1,1.1 \%$ CRF02_AG/A1, $0.5 \%$ CRF37, $0.5 \%$ CRF13, $0.5 \% \quad \mathrm{C}, \quad 0.5 \%$ CRF02_ AG/CRF01_AE, $0.5 \%$ CRF01_AE/G) (data not shown). With the exception of the ViroSeq ${ }^{\mathrm{TM}}$ positive standard, which was sequenced using both assays, there was no case of subtype B in the study population.

Sequencing performance of ViroSeq ${ }^{\mathrm{TM}}$

and the in-house assay

As shown in Table 1, 98.7\% of the 156 samples processed using the in-house protocol were successfully sequenced, with $64.1 \%$ of the amplicons obtained with the first-round RT-PCR and the remaining $35.9 \%$ after a semi-nested PCR. For the ViroSeq ${ }^{\mathrm{TM}}$ assay, amplification 
Table 1 Detailed sequencing results for the two genotyping assays: in-house and ViroSeq ${ }^{\text {TM }}$

\begin{tabular}{lll}
\hline Sequencing evaluation & In-house assay & ViroSeq $^{\mathrm{TM}}$ assay \\
\hline Number of samples processed & 156 & 76 \\
RT-PCR & 105 & 71 \\
Semi-nested-PCR & 49 & Not applicable \\
Sequencing performance, N (\%) & $154(98.7 \%)$ & $70(92.1 \%)$ \\
\hline
\end{tabular}

was effective for 70 out of $76(92.1 \%)$ samples processed with this commercial kit, whose amplification threshold range is 3.3-5.87 $\log _{10}$ RNA copies/ml (Celera Diagnostic, USA 2007).

Of the six amplified samples that were not using ViroSeq ${ }^{\mathrm{TM}}$, five were successfully processed with the in-house assay, and phylogeny revealed subtypes A1 (2 samples, plasma viral load 4.35 and $4.85 \log _{10}$ RNA copies/ml), D (2 samples, plasma viral load 4.05 and $5.42 \log _{10}$ RNA copies/ml), and CRF02_AG (1 sample, plasma viral load $1.79 \log _{10}$ RNA copies/ml, which was below ViroSeq $^{\mathrm{TM}}$ amplification threshold). Thus, amplification by ViroSeq $^{\mathrm{TM}}$ was ineffective for subtypes A1 and D.

The range of viremia for the samples processed by the in-house method was identical to that of samples processed with ViroSeq ${ }^{\mathrm{TM}}$ (1.79-6.96 $\log _{10}$ RNA copies/ml), avoiding bias when comparing the amplification performance of the two methods.

An overview of the ViroSeq ${ }^{\mathrm{TM}}$ sequencing primers showed a good alignment with all the sequences, with the only exception being primer $\mathrm{D}$, which aligned to 10 out of $70(14.2 \%)$ sequences obtained with the ViroSeq ${ }^{\mathrm{TM}}$ system. The seven "in-house" sequencing primers also showed a good alignment to the sequences, without any significant mismatch.
Comparative analysis of the mutation profiles obtained with the in-house and Viroseq ${ }^{\mathrm{TM}}$ assays

To compare the mutation profiles obtained by the two genotyping assays, 72 sequences (complete sequence length $\approx 1,197 \mathrm{bp}$ ) generated from the 36 samples processed with both assays (in-house and ViroSeq ${ }^{\mathrm{TM}}$ ) were analyzed. Each of the samples was from a different subject. A total of 14,364 amino acid positions $([99 \mathrm{PR}+300 \mathrm{RT}] \times 36$ pairs of samples) were analyzed for a comparative evaluation between the ViroSeq ${ }^{\mathrm{TM}}$ and the in-house assays. The rate of discordance between the two assays was negligible (PR region: $0.17 \%$ [6/3,564]; RT region $0.27 \%[29 / 10,800])$, with an overall discordance value of $0.24 \%(35 / 14,364)$ in the entire analysed region. The overall concordance considering both the PR and RT regions was $99 \%(14,226 /$ 14,364). No indeterminate results were observed.

Analysis dedicated to the identification of drug-resistance-associated amino acid positions revealed a very high degree of concordance for the two methods. Specifically, among the 612 drug resistance positions analyzed in the PR region (17 amino acid positions $\times 36$ pairs of samples), no discordance was observed, and only three mutations $(0.49 \%)$ were partially concordant in three different patients: two at position 54 and one at position 76 (Table 2). On the other hand, among the $864 \mathrm{RT}$ drug resistance positions analyzed ( 24 amino acid positions $\times 36$ pairs of samples), a low degree of discordance $(3 / 864,0.35 \%)$ was observed at positions 74 , 101 and 103. These discrepancies in the RT mutational profile were found in three patients. The rate of partial concordance between the two methods was $0.57 \%$ (5/864), found in four different patients at drug resistance position 69,70 and 75 . Interestingly, in all the cases of partial concordance, ViroSeq ${ }^{\mathrm{TM}}$ detected more viral mixtures than the in-house protocol. Analysis of drug resistance-associated positions in both $(\mathrm{PR}+\mathrm{RT})$ regions $(1476 ; 41$ amino acid
Table 2 Discordant and partially concordant mutations detected in drug-resistanceassociated positions

\begin{tabular}{lllll}
\hline Category & Gene region & Wild type & \multicolumn{2}{l}{ Detected aa mutations } \\
\cline { 5 - 5 } & & aa position & ViroSeq $^{\mathrm{TM}}$ kit & In-house protocol \\
\hline Partial concordance & Protease & I54 & IM & I \\
& & I54 & IF & I \\
& \multirow{2}{*}{ Reverse transcriptase } & L76 & VL & L \\
& & K70 & NS & KE \\
& & V75 & AV & K \\
& & V75 & ILV & V \\
Discordance & V75 & MV & V \\
& \multirow{2}{*}{ Reverse transcriptase } & L74 & V & L \\
& & K101 & P & E \\
& & K103 & N & R \\
\hline
\end{tabular}


positions $\times 36$ pairs of samples) revealed a very high degree of concordance, $99.25 \%$ (1465/1476), with no evidence of indeterminate results.

Evaluation of the pairwise genetic distances between sequence pairs obtained by both methods showed a high similarity in the nucleotide outputs. The mean genetic distance between sequence pairs was 0.0003 for the PR region (ranging from 0 to 0.057 ) and 0.0002 for the RT region (ranging from 0 to 0.042 ).

Phylogenetic analysis confirmed that sequences of each sample generated with both assays clustered together with a high $(>90 \%)$ bootstrap robustness, thus demonstrating homology in subtyping and fidelity in the reproducibility of the two protocols (Fig. 1). Only a single sequence pair was not found to cluster together monophyletically using the bioinformatics tools used for phylogenetic tree construction.

Cost evaluation of the in-house assay

A cost analysis was performed to evaluate the financial aspect potentially related to the use of the in-house protocol for monitoring drug-resistant HIV-1 strains. The overall cost per sample using this protocol was around $92 €$ compared to $217 €$ with ViroSeq ${ }^{\mathrm{TM}}$, despite the common consumables used in both protocols (Table 3). Coupled with its reliability and validity, as shown above, the low cost of this in-house protocol will therefore reduce to about half the cost for HIV genotyping, compared to the ViroSeq ${ }^{\mathrm{TM}}$ kit. This alternative could increase accessibility to surveillance of HIV-1 drug resistance in the health systems as well as access to routine clinical practices for medium to large population-based genotyping services, both in resource-limited and high-viral-genetic-diversity settings.

\section{Discussion}

With the increasing availability of generic HAART drugs in Cameroon, the establishment of an accurate, reproducible, and highly sensitive but also affordable assay for monitoring viral evolution and drug resistance of emerging strains in patients undergoing treatment is becoming a

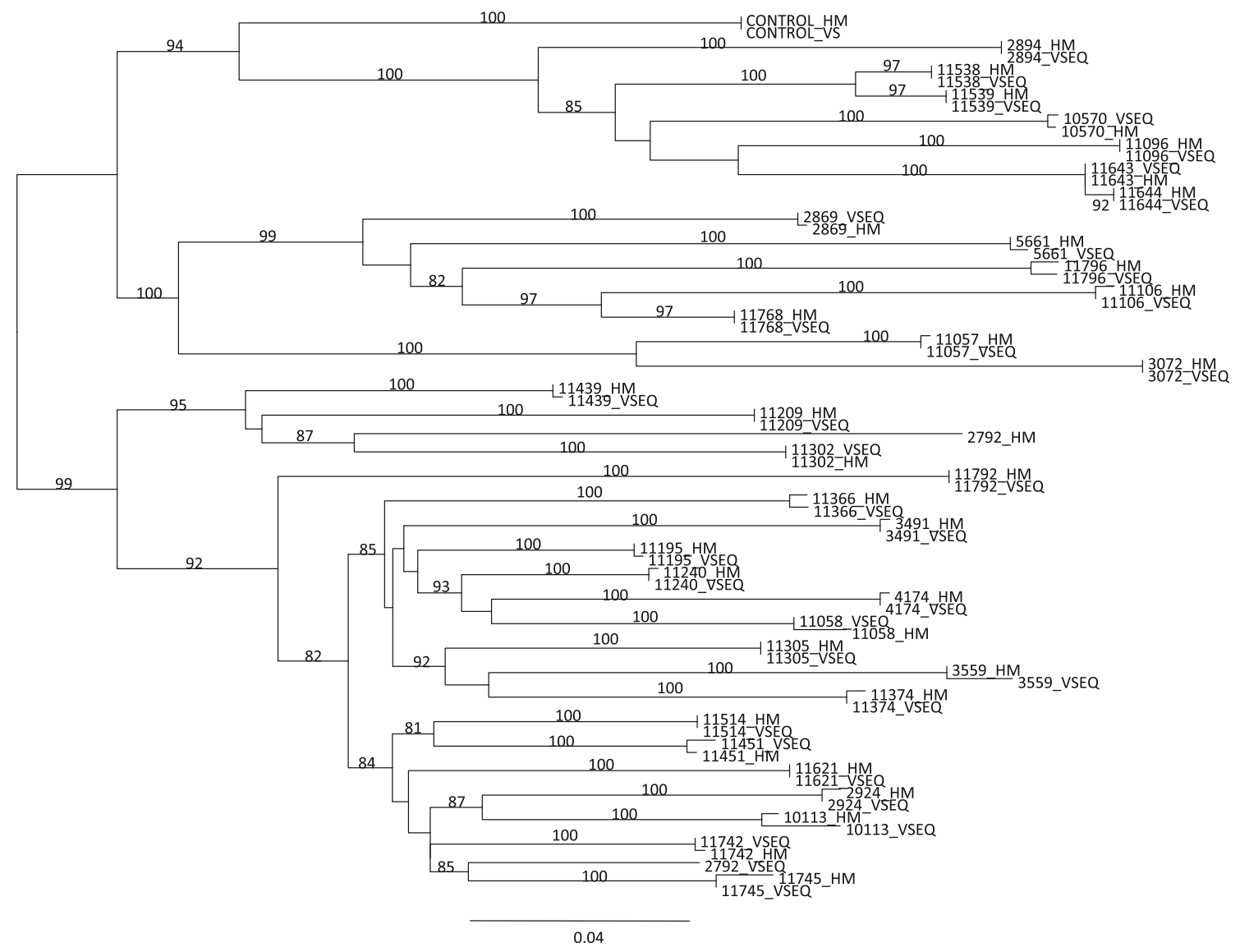

Fig.1 Maximum-likelihood phylogenetic analysis of $72 \mathrm{HIV}-1$ pol gene sequences generated from 36 pairs of samples. Sequences labeled as "HM" were obtained with the Home Made (in-house) sequencing protocol, while sequences marked as "VSEQ" were obtained with the ViroSeq ${ }^{\mathrm{TM}}$ assay. The scale at the bottom indicates
0.04 substitutions/site. Numbers above branches indicate the statistical robustness and reliability of the branching order, estimated with bootstrap support using 1000 replicates on a maximum-likelihood tree. Only bootstrap values $>80$ are shown on the tree 
Table 3 Comparative cost analysis between the $\operatorname{ViroSeq}^{\mathrm{TM}}$ kit and the in-house protocol for HIV-1 genotyping

\begin{tabular}{|c|c|c|c|c|}
\hline \multirow[t]{3}{*}{ Cost of each sequencing step } & \multicolumn{4}{|c|}{ HIV-1 genotyping costs for each patient } \\
\hline & \multicolumn{2}{|l|}{ ViroSeq $^{\mathrm{TM}}$ kit } & \multicolumn{2}{|c|}{ In-house assay } \\
\hline & Euro & Franc CFA & Euro & Franc CFA \\
\hline RNA extraction (QIAGEN) & 5 & 3,280 & 5 & 3,280 \\
\hline RT-PCR ViroSeq ${ }^{\mathrm{TM}}$ reagents (Applied Biosystems) & 195 & 127,920 & Not required & - \\
\hline RT-PCR in-house reagents (Invitrogen) & Not required & - & 11 & 7,216 \\
\hline AmpliTaqGold for nested PCR (Applied Biosystems) & Not required & - & 3 & 1,968 \\
\hline PCR product purification (Millipore column) & 2.50 & 1,640 & 2.50 & 1,640 \\
\hline Electrophoresis visualization cost (DNA ladder, loading buffer) & 2 & 1,312 & 2 & 1,312 \\
\hline Sequencing reaction (Big Dye Terminator; Applied Biosystem) & Not required & - & 56 & 36,736 \\
\hline Purification of sequencing products & 7 & 4,592 & 7 & 4,592 \\
\hline Costs for genetic analyzer usage & 6 & 3,936 & 6 & 3,936 \\
\hline Total cost for each HIV-1 pol sequence & 217.50 & 142,680 & 92.50 & 60,680 \\
\hline
\end{tabular}

This estimate was performed on the basis of the current costs (2009) of reagents in Cameroon, expressed both in Euro and Central African Franc

public-health priority. Indeed, accessibility to antiretroviral drugs without adequate monitoring may result in the rapid spread of drug-resistant HIV-1 strains. The in-house assay developed in this study represents a good alternative to the standard commercially available HIV-1 genotyping assays by reducing costs while maintaining the same specificity and sensitivity in detecting HIV-1 drug resistance mutations. More importantly, the in-house protocol reported here was found to have a high PCR sensitivity, with a large number of amplicons already produced in the first PCR step $(64.1 \%)$. Furthermore, it was also able to sequence $98.7 \%$ of plasma samples with detectable viremia, even at very low values (1.79 $\log _{10}$ RNA copies/ml), due to the use of a sensitive semi-nested PCR.

These findings, in terms of reproducibility and capability of detecting drug-resistance-associated mutations, are similar to data reported by Saravanan et al. [24]. Particularly, drug-resistance mutations associated with treatment failure were identified perfectly using the $\operatorname{ViroSeq}^{\mathrm{TM}}$ kit and our in-house assay. Discordant mutations at drugresistance-associated positions were identified only in less than $0.3 \%$ of the overall studied population; their presence likely reflects the normal test-to-test variability or the prevalence of quasispecies [41]. An almost full mutational similarity between the pairs of sequences was obtained by both assays (the commercial and the in-house) in all 399 analysed amino acids encompassing the PR and RT regions (and not only restricted to drug resistance positions).

The in-house method has been shown to identify a wide spectrum of HIV-1 strains belonging to different subtypes (7 pure subtypes, 5 known CRFs, and 4 rare unclassified recombinant forms), using specific primers matching in highly conserved regions. The capacity to identify these subtypes was greater compared to other in-house antiretroviral drug resistance assays used previously in limited-resource settings. These previous in-house assays were restricted either to the sequencing of four pure subtypes (A1, B, C, D) and two known CRFs (CRF01_AE and CRF02_AG) in South Africa [25] or to three pure subtypes (B, C, D) in the case of the Indian study [24]. Therefore, the results indicate a highly successful sequencing performance using the in-house protocol in a context of high HIV-1 genetic diversity, as is found in sub-Saharan Africa [42, 43].

Although sequence editing was more laborious and time-consuming with the in-house method, both protocols had very similar laboratory and usage characteristics. For instance, the required time scales for completion of both assays were quite similar (ViroSeq ${ }^{\mathrm{TM}}, 16$ hours; in-house assay, 20 hours), with the second step PCR (semi-nested) constituting the time difference between the two methods. Therefore, personnel should be well trained to ensure appropriate analysis and a relevant resistance report with this in-house protocol.

Moreover, despite the high risk of cross-contamination with nested PCR [44], results obtained in this study showed no event of this nature. Therefore, this finding proves that compliance with these laboratory criteria is a sufficient approach to ensure valid and reliable results in a standard molecular biology laboratory [45]. A contamination check also excluded the possibility of sample mix-up, thus suggesting that the unpaired sequences from the same sample were possibly due to an inaccurate identification of recombination using the bioinformatics tools.

Finally, it should be emphasized that the in-house assay costs approximately $50 \%$ less $(92 €)$ than the ViroSeq ${ }^{\mathrm{TM}}$ kit $(217 €)$, a cost that appears similar to the one reported with the in-house HIV-1 genotyping system developed by 
Saravanan et al. [24] ( $\$ 100$ vs. $\$ 230$ for $\left.\operatorname{ViroSeq}^{\mathrm{TM}}\right)$. Therefore, the use of this low-cost assay can significantly improve the management of HIV-1 infection in countries with limited resources for medium and large populationbased genotyping services, representing a useful weapon against the spread of HIV-resistant strains [46].

Further studies spanning a wider spectrum of viral loads, subtypes and known resistant HIV-1 isolates are necessary to better investigate the analytical sensitivity of this inhouse sequencing assay for the detection of drug resistance mutations.

In conclusion, the present study describes the validation of a new in-house HIV-1 genotyping system for plasma samples. The wider coverage of HIV-1 genetic diversity, together with its low cost, makes this in-house assay a suitable and affordable tool for resource-limited settings. Moreover, it represents an attractive alternative for HIV-1 drug resistance surveillance and for reducing the risk of selection of further drug-resistance-associated mutations in financially constrained public health systems.

Acknowledgments This work was supported financially by grants from the Italian National Institute of Health, Rome, through funding from the Italian Cooperation to the government of Cameroon, and by the European Commission Framework 7 Programme (CHAIN, the Collaborative HIV and Anti-HIV Drug Resistance Network, Integrated Project no. 223131). We would like to thank Prof Lutz Gürtler for his precious contributions in the revision of the manuscript.

\section{References}

1. World Health Organization (2009) Aids epidemic updates December 2009

2. WHO/UNAIDS (2003) The WHO and UNAIDS global initiative to provide antiretroviral therapy to 3 million people with HIV/ AIDS in developing countries by the end of 2005. http://www.whoint/hiv

3. World Health Organization (2006) Antiretroviral therapy for HIV infection in adults and adolescents in resource-limited settings: towards universal access: recommendations for a public health approach. http://wwwwhoint/hiv/pub/guidelines/adult/en/index html

4. Braitstein P, Brinkhof MW, Dabis F, Schechter M, Boulle A, Miotti P, Wood R, Laurent C, Sprinz E, Seyler C, Bangsberg DR, Balestre E, Sterne JA, May M, Egger M (2006) Mortality of HIV1-infected patients in the first year of antiretroviral therapy: comparison between low-income and high-income countries. Lancet 367:817-824

5. Bennett DE (2006) The requirement for surveillance of HIV drug resistance within antiretroviral roll out in the developing world. Curr Opin Infect Dis 19:607-614

6. European Guidelines Group for HIV Resistance (2009) Clinical and laboratory guidelines for the use of HIV-1 drug resistance testing as part of treatment management: recommendations for the European setting. AIDS 15:309-320

7. Panel on Antiretroviral Guidelines for Adults and Adolescents Guidelines for the use of antiretroviral agents in HIV-1-infected adults and adolescents. Department of Health and Human Services
December 1, 2009, pp 1-161. http://www.aidsinfonihgov/ ContentFiles/AdultandAdolescentGL.pdf

8. Laurent C, Kouanfack C, Koulla-Shiro S, Njoume M, Nkene YM, Ciaffi L, Brulet C, Peytavin G, Vergne L, Calmy A, MpoudiNgole E, Delaporte E (2007) Long-term safety, effectiveness and quality of a generic fixed-dose combination of nevirapine, stavudine and lamivudine. AIDS 21:768-771

9. Boyer S, Eboko F, Camara M, Abé C, Nguini ME, Koulla-Shiro S, Moatti JP (2010) Scaling up access to antiretroviral treatment for HIV infection: the impact of decentralization of healthcare delivery in Cameroon. AIDS 24(Suppl 1):S5-S15

10. Ndongmo CB, Pieniazek D, Holberg-Petersen M, Holm-Hansen C, Zekeng L, Jeansson SL, Kaptue L, Kalish ML (2006) HIV Genetic diversity in Cameroon: possible public health importance. AIDS Res Hum Retroviruses 22:812-816

11. Vallari A, Bodelle P, Ngansop C, Makamche F, Ndemb N, Mbanya D, Kaptue L, Gurtler L, McArthur C, Devare S (2010) Four new HIV-1 group $\mathrm{N}$ isolates from Cameroon: prevalence continues to be low. AIDS Res Hum Retroviruses 26:109-115

12. Plantier JC, Leoz M, Dickerson JE, de Oliveira F, Cordonnier F, Lemée V, Damond F, Robertson DL, Simon F (2009) A new human immunodeficiency virus derived from gorillas. Nat Med $15: 871-872$

13. Comité National de lutte contre le SIDA, Ministère de la Santé Publique du Cameroun July 2009 Profil des estimations pays du VIH SIDA au Cameroun

14. Bennett DE, Camacho RJ, Otelea D, Kuritzkes DR, Fleury H, Kiuchi M, Heneine W, Kantor R, Jordan MR, Schapiro JM, Vandamme AM, Sandstrom P, Boucher CA, van de Vijver D, Rhee SY, Liu TF, Pillay D, Shafer RW (2009) Drug resistance mutations for surveillance of transmitted HIV-1 drug-resistance: 2009 update. PLoS One 4(3):e4724

15. Gallant JE (2006) Antiretroviral drug resistance and resistance testing. Top HIV Med 13:138-142

16. Johnson VA, Brun-Vezinet F, Clotet B, Gunthard HF, Kuritzkes DR, Pillay D, Schapiro JM, Richman DD (2009) Update of the drug resistance mutations in HIV-1. Top HIV Med 63:585-592

17. Tommy F, Liuand R, Shafer W (2006) Web resources for HIV type 1 genotypic resistance test interpretation. Clin Infect Dis 42:1608-1618

18. Mukaide M, Sugiura W, Matuda M, Usuku S, Noguchi Y, Suzuki K, Kawata K, Ito A, Sagara H, Yamada K, Kondo M, Imai M (2000) Evaluation of ViroSeq ${ }^{\mathrm{TM}}$ HIV version 2 for HIV drug resistance. Jpn J Infect Dis 53:203-205

19. Mracna M, Becker-Pergola G, Dileanis J, Guay LA, Cunningham S, Jackson JB, Eshleman SH (2001) Performance of the applied biosystems ViroSeq ${ }^{\mathrm{TM}}$ HIV-1 genotyping system for sequencebased analysis of non-subtype b HIV-1 from Uganda. J Clin Microbiol 39:4323-4327

20. Eshleman SH, Hackett J Jr, Swanson P, Cunningham SP, Drews B, Brennan C, Devare SG, Zekeng L, Kaptue L, Marlowe N (2004) Performance of the Celera Diagnostics ViroSeq ${ }^{\mathrm{TM}}$ HIV-1 genotyping system for sequence-based analysis of diverse human immunodeficiency virus type 1 strains. J Clin Microbiol 42:2711-2717

21. Ribas SG, Leo H, Pascale O, Katrien F (2006) Performance evaluation of the two protease sequencing primers of the Trugene HIV-1 genotyping kit. J Virol Methods 135:137-142

22. Clavel F, Hance AJ (2004) HIV drug resistance. N Engl J Med 350:1023-1035

23. Engelbrecht S, VanZyl G, Claassen M, Laten A, Jacobs G, Preiser W (2007) Sensitive in-house RT-PCR assay for the assessment of HIV-1 antiretroviral drug resistance. SA-AIDS, p 528

24. Saravanan S, Vidyaa M, Balakrishanana P, Kumarasamya N, Solomona SS, Solomona S, Kantor R, Katzensteinc D, Ramratnamb B, Mayer KH (2009) Evaluation of two human 
immunodeficiency virus-1 genotyping systems: ViroSeq ${ }^{\mathrm{TM}} 20$ and an in-house method. J Virol Methods 159:211-216

25. Wallis CL, Papathanasopoulos MA, Lakhi S, Karita E, Kamali A, Kaleebu P, Sanders E, Anzala O, Bekker LG, Stevens G, de Wit TF, Stevens W (2010) Affordable in-house antiretroviral drug resistance assay with good performance in non-subtype B HIV-1. J Virol Methods 163:505-508

26. Lee S, Espin F, Turner J, Griswold M, Kahn D (1999) Genotyping of HIV-1 drug-resistance: comparison between in-house procedure and visible genetics Trugene [Tm] HIV-1 assay. Abstr Intersci Conf Antimicrob Agents Chemother 39:462

27. Loubsher S, Sherman G, Chezzi C, Jones J, Cohen S, Puren A, Morris L (2004) Characterization of nevirapine resistance mutations using RT-PCR and DNA sequencing methods in a motherinfant cohort following single dose nevirapine. Antivir Ther 9:S145

28. Steegen K, Demecheleer E, De Cabooter N, Nges D, Temmerman M, Ndumbe P, Mandaliya K, Plumb J, Verhofstede C (2006) A sensitive in-house RT-PCR genotyping system for combined detection of plasma HIV-1 and assessment of drug resistance. J Virol Methods 133:137-145

29. Lindstrom A, Albert J (2003) A simple and sensitive in-house method for determining genotypic drug resistance of HIV-1. J Virol Methods 107:45-51

30. Laurent C, Kouanfack C, Vergne L, Tardy M, Zekeng L, Noumsi N, Butel C, Bourgeois A, Mpoudi-Ngole E, Koulla-Shiro S, Peeters M, Delaporte E (2006) Antiretroviral drug resistance and routine therapy. Cameroon Emerg Infect Dis 12:1001-1004

31. Aghokeng FA, Vergne L, Mpoudi NE, Mbangue M, Deoudje N, Mokondji E, Nambei WS, Peyou-Ndi MM, Moka JJL, Delaporte E, Peeters M (2009) Evaluation of transmitted HIV drug resistance among recently-infected antenatal clinic attendees in four Central African countries. Antiviral Therapy 14:401-411

32. Kouanfack C, Montavon C, Laurent C, Aghokeng A, Kenfack A, Bourgeois A, Koulla-Shiro S, Mpoudi-Ngole E, Peeters M, Delaporte E (2009) Low levels of antiretroviral-resistant HIV infection in a routine clinic in Cameroon that uses the World Health Organization (WHO) public health approach to monitor antiretroviral treatment and adequacy with the WHO recommendation for second-line treatment. Clin Infect Dis 48:1318-1322

33. Hall T (2001) BioEdit version 506. Department of Microbiology, North Carolina State University, copyright 1997-2001. Last updated on $2 / 12 / 2001$

34. Swofford DL (2002) PAUP phylogenetic analysis using parsimony (*and other methods): version 40. Sinauer Associates, Sunderland
35. Posada D, Buckley TR (2004) Model selection and model averaging in phylogenetics: advantages of akaike information criterion and bayesian approaches over likelihood ratio tests. Syst Biol 53:793-808

36. Guindon S, Gascuel O (2003) A simple, fast, and accurate algorithm to estimate large phylogenies by maximum likelihood. Syst Biol 52:696-704

37. Kosakovsky PSL, Posada D, Stawiski E, Chappey C, Poon AF, Hughes G, Fearnhill E, Gravenor MB, Leig BAJ, Frost SD (2009) An evolutionary model-based algorithm for accurate phylogenetic breakpoint mapping and subtype prediction in HIV-1. PLoS Comput Biol 5(11):e1000581

38. Struck D, Perez BD, Devaux C, Schmit JC (2010) COMET: a novel approach to HIV-1 subtype prediction. 8th European HIV drug resistance workshop. Abstract 88

39. Lole KS, Bollinger RC, Paranjape RS, Gadkari D, Kulkarni SS, Novak NG, Ingersoll R, Sheppard HW, Ray SC (1999) Fulllength human immunodeficiency virus type 1 genomes from subtype C-infected seroconverters in India, with evidence of intersubtype recombination. J Virol 73:152-160

40. Maes B, Schrooten Y, Snoeck J, Derdelinckx I, Van Ranst M, Vandamme AM, van Laethem K (2004) Performance of ViroSeq HIV-1 genotyping system in routine practice at a Belgian clinical laboratory. J Virol Methods 119:45-49

41. Diana DH, Susan HE, Donald JB, Paul EP, James WB (2003) Evaluation of the editing process in human immunodeficiency virus type 1 genotyping. J Clin Microbiol 41:3265-3272

42. Peeters M, Toure-Kane C, Nkengasong JN (2003) Genetic diversity of HIV in Africa: impact on diagnosis, treatment, vaccine development and trials. AIDS 17:2547-2560

43. Butler IF, Pandrea I, Marx PA, Apetrei C (2007) HIV genetic diversity: biological and public health consequence. Curr HIV Res 5:23-45

44. Mitchell C, Kraft K, Peterson D, Frenkel L (2010) Cross-contamination during processing of dried blood spots used for rapid diagnosis of HIV-1 infection of infants is rare and avoidable. J Virol Methods 163:489-491

45. Duchassaing D, Dingeon B, Chalas J, Coude M, Coulhon MP, Launay JM (2002) Molecular genetics: pre- and post-analytic "best practices". Ann Biol Clin (Paris) 60:617-621

46. Hamers RL, Derdelinckx I, van Vugt M, Stevens W, Rinke de Wit TF, Schuurman R (2008) The status of HIV-1 resistance to antiretroviral drugs in sub-Saharan Africa. Antivir Ther $13: 625-639$ 